\title{
Formation of Plate-Like Apatite Coatings on Polymer Surface
}

\author{
Y. F. Chou ${ }^{1}$, W.-A. Chiou ${ }^{2}$, and B. M. Wu ${ }^{1,3}$ \\ ${ }^{1}$ Dept. of Bioengineering, University of California, Los Angeles, CA, 90095 \\ 2 Dept. of Chemical Eng. \& Materials Science, University of California, Irvine, CA, 92697 \\ ${ }^{3}$ Dept. of Materials Science \& Engineering, University of California, Los Angeles, CA, 90095
}

Apatite coated polymer scaffolds have been investigated extensively for bone engineering. Apatite-induced in vitro mineralization and in vivo bone formation have been reported [1]. Several apatite structures have been observed during various stages of apatite formation, each with distinct morphology, composition, and corresponding MC3T3-E1 osteoblast attachment efficiency, cell morphology and cytoskeletal arrangement [2]. While apatite coating on various substrates have been achieved, the detailed growth mechanism of apatite remains unclear. In fact, the interfacial morphology of the apatite-substrate interface is not fully established. The objective of this paper is to describe the apatite-polymer interface using cross-sectional TEM.

Polystyrene culture dishes (Corning) were argon plasma-etched, and immersed in concentrated simulated body fluid ( $5 \mathrm{x} \mathrm{SBF}$ ) that resembles five times human blood plasma ionic concentration for 24 hours. The solution was then changed to $\mathrm{Mg}^{2+}$ and $\mathrm{HCO}_{3}^{-}$free 5x SBF for 2 days [3,4]. The entire process was at $37^{\circ} \mathrm{C}$. After initial examination of surface morphology in a SEM, apatite coated polystyrene dish samples were trimmed to elongated triangular shape and put into a BEEM capsule filled with LR White embedding medium. Ultra-thin cross sections were cut with a diamond knife on a Sorvall MT-2 ultramicrotome and coated with a thin layer of carbon to preserve the integrity of ultra-thin sections. The internal and interfacial structures of the apatite-polymer interface were then examined with cross-sectional SEM and a TEM.

A backscattered electron (BSE) reveals a distinct apatite layer (6-7 $\mu \mathrm{m}$ in thickness) on the polystyrene substrate (Fig. 1). The EDS confirms the calcium and phosphate content of the typical apatite (Fig. 2). Although both higher magnification BSE and SEM images of the apatite layer depict apatite crystals are plate-like in nature (Figs. 2 and 3), images at the interface were rather unclear. Nevertheless, the morphology of apatite was clearly depicted in TEM images throughout the layer (Figs. 4 and 5). A low magnification TEM image (Fig. 4) reveals a fair amount of porosity within the apatite layer. A thin apatite layer $(\sim 0.3 \mu \mathrm{m}$, arrows in Fig. 4$)$ comprised of many minute and elongated needles at the interface is also observed. Higher magnifications TEM lattice image (open arrows) and electron diffraction indicate those needle-like crystals were apatite (Fig. 6), in agreement of previous XRD studies [2]. The low contrast, somewhat rounded structures in Fig. 5 and 6 are thought to be thin platy apatite crystals that were cut parallel or tangential to the a- or c-axis (arrows in Fig. 5 and 6). The thickness of apatite platelets ranges from 3 to nearly $100 \mathrm{~nm}$ indicating the different stages of crystal growth. While apatite crystals at the interface are much smaller in size and thickness, some rather large/thick platelets can be seen in the middle and upper parts of the coating. Whether the apatite was initially crystallized as needle/fine platelets, or transformed from rounded amorphous precursors, will be investigated by characterizing the interface at various early time points of apatite formation.

\section{References}

[1] I. Manjubala, et al., J. Biomed. Mater. Res., 63 (2) (2002) 200.

[2] I. Wulur. M.S. Thesis, UCLA Department of Bioengineering (2002)

[3] M. Tanahashi et al., J. Am. Ceram. Soc., 77 (11) (1994) 2805.

[4] F. Barrere et al., Biomaterials. 23 (9) (2002) 1921. 


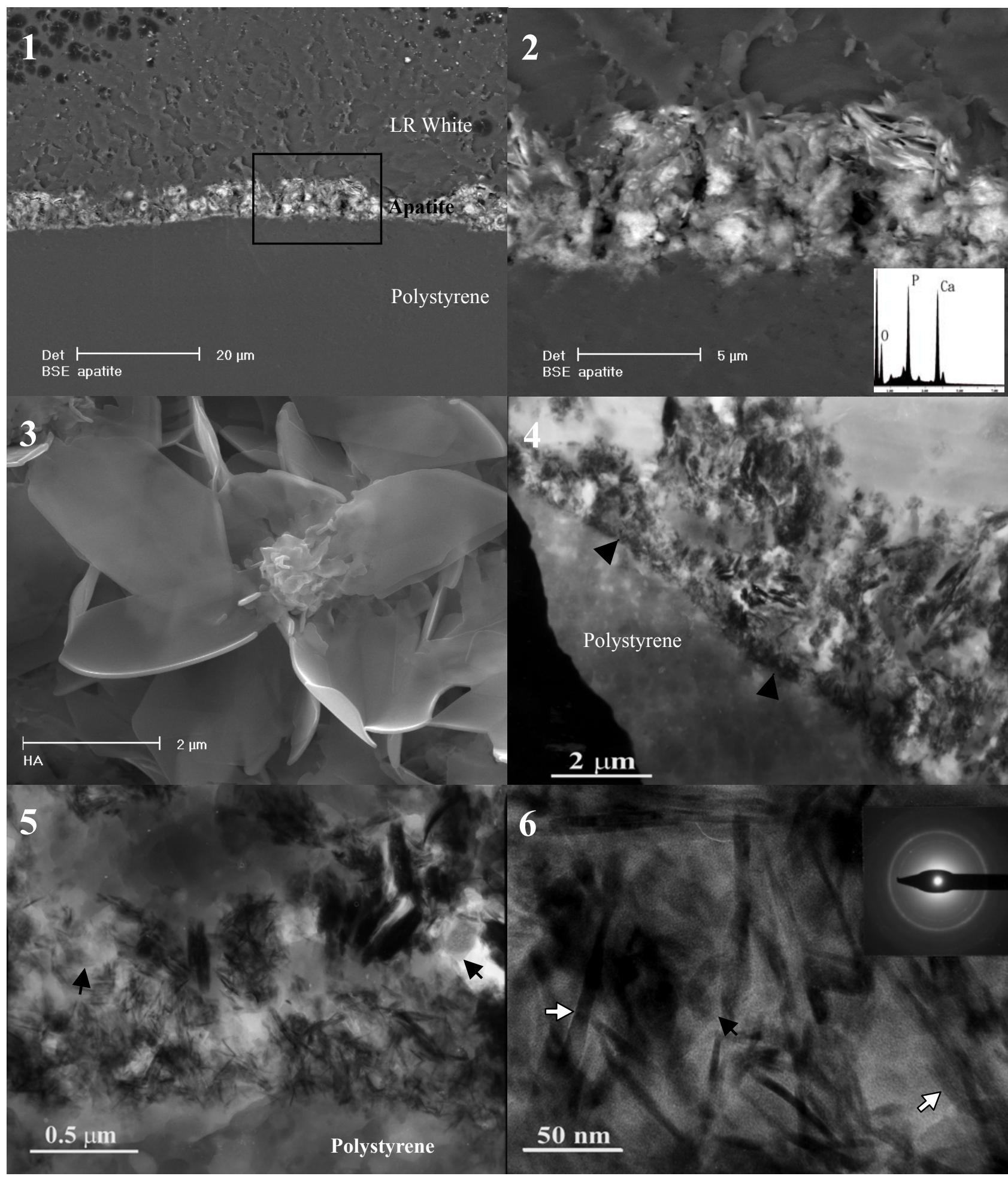

Fig. 1. Back scattering electron (BSE) image of the ultramicrotomed cross-section

Fig. 2. A close-up view of BSE image and EDS of the ultra-thin cross-section

Fig. 3. SEM image of plate-like apatite coating showing somewhat rounded platy morphology

Fig. 4. TEM image of the ultra-thin cross-section depicting the apatite layer

Fig. 5. TEM image of the apatite layer at the interface revealing the very minute needle/platelets

Fig. 6. Higher magnification TEM image and diffraction pattern demonstrating the nano- crystalline nature of apatite minerals 